\title{
Prognostic Value of Myocardial Perfusion SPECT After Intravenous Bolus Administration of Nicorandil in Patients with Acute Ischemic Heart Failure
}

\author{
Yoshimitsu Fukushima ${ }^{1}$, Shin-ichiro Kumita ${ }^{1}$, Yukichi Tokita², and Naoki Sato ${ }^{2}$ \\ ${ }^{1}$ Department of Radiology, Nippon Medical School Hospital, Tokyo, Japan; and ${ }^{2}$ Department of Cardiovascular Medicine, Nippon \\ Medical School Hospital, Tokyo, Japan
}

\begin{abstract}
Nicorandil, a hybrid adenosine triphosphate (ATP)-sensitive potassium channel opener and nitrate, is commonly used for the management of acute ischemic heart failure (AlHF). The aims of this study were to predict the effect of nicorandil by calculating myocardium-tobackground ratio increasing rate (MBR-IR) using nicorandil stress myocardial perfusion SPECT and to evaluate the prognostic value of MBR-IR in patients with AlHF. Methods: Twenty-two patients (age, $70 \pm 12 \mathrm{y}$ ) admitted to the coronary care unit with AlHF underwent nicorandil-stress and rest myocardial perfusion SPECT. Using these images, MBR-IR was calculated by dividing stress MBR by rest MBR (MBR = peak value of left ventricular myocardial segments/mean value of upper mediastinum). In order to evaluate the clinical importance of MBR-IR derived from the nicorandil-stress test, all patients were divided into 2 groups, based on the value of MBR-IR. All patients were observed over $5 \mathrm{y}$ from the onset of AlHF for the occurrence of major adverse cardiac events (MACE). Results: Both high- and low-MBR-IR groups contained 11 participants. Median MBR-IR was 1.55 (1.34-1.61) in the high-MBR-IR group and $1.08(1.02-1.10)$ in the low-MBR-IR group. The proportion of patients who experienced MACE was significantly higher in the low-MBRIR group than in the high-MBR-IR group ( $91 \%$ vs. $18 \%, P<0.001)$. Conclusion: This study demonstrated that the MBR-IR calculated using nicorandil-stress myocardial perfusion SPECT may have a high prognostic value for MACE in patients with AlHF.
\end{abstract}

Key Words: acute ischemic heart failure; nicorandil-stress myocardial perfusion imaging; single photon emission computed tomography; myocardium-to-background ratio increasing rate; major adverse cardiac event

J Nucl Med 2016; 57:385-391

DOI: 10.2967/jnumed.115.162420

\section{A}

cute ischemic heart failure (AIHF) is often associated with multivessel coronary artery disease (1). The patients usually have a long history of revascularization treatments, including percutaneous coronary intervention and coronary artery bypass grafting, and thus additional revascularization procedures are not preferred. This often leads to a reliance on medical therapy to maintain their

For correspondence or reprints contact: Yoshimitsu Fukushima, Department of Radiology, Nippon Medical School, 1-1-5, Sendagi, Bunkyo-ward, Tokyo 113-8603, Japan.

E-mail: fuku@nms.ac.jp

Published online Dec. 3, 2015.

COPYRIGHT (C 2016 by the Society of Nuclear Medicine and Molecular Imaging, Inc. clinical condition $(2,3)$. Nitrates are commonly used for the management of AIHF in the acute phase (4-7). Nicorandil ( $N$-(2-hydroxyethyl)-nicotinamide nitrate; Chugai), a hybrid of an adenosine triphosphate (ATP)-sensitive potassium channel opener and a nitrate, is also used to treat patients with AIHF to improve the recovery of postischemic cardiac contractile function $(8,9)$. Nicorandil exerts a vasodilatory effect on the systemic veins, and it also dilates arteries, including the peripheral arteries, by opening ATPsensitive potassium channels (10-12). Previous studies have shown that the intravenous administration of nicorandil in the acute phase of AIHF improves cardiac output, reduces pulmonary pressure, and modulates hemodynamic parameters $(13,14)$.

Traditionally, vasodilators have been used to calculate both myocardial flow reserve (MFR) and fractional flow reserve (FFR) (15). However, recent studies have found nicorandil to be a safer and more effective vasodilator than adenosine for the measurement of FFR $(16,17)$. Therefore, nicorandil may also be used to estimate MFR. MFR is commonly measured by myocardial perfusion PET/CT and provides insight on the severity of coronary artery disease $(18,19)$. MFR calculation using conventional SPECT has not been possible until now. Myocardial blood flow increasing rate (MBF-IR), derived from myocardial perfusion SPECT using dynamic acquisition similar to PET/CT, could be an alternative to coronary flow reserve (CFR) (20). However, there is no established alternative method for calculating CFR using conventional, nondynamic myocardial perfusion SPECT.

The aims of this study were to assess the effect of nicorandil by calculating myocardium-to-background ratio increasing rate (MBR-IR) as an alternative to CFR using conventional myocardial perfusion SPECT with intravenous administration of nicorandil and to evaluate the prognostic value of MBR-IR in patients with AIHF.

\section{MATERIALS AND METHODS}

\section{Patient Population}

Thirty consecutive patients with suspected AIHF, admitted to the coronary care unit between November 2008 and October 2009, were considered for this study. The diagnoses were based on serum myocardial enzyme activity, electrocardiogram, transthoracic echocardiography, and coronary angiography (CAG) findings. Eight patients with nonischemic heart failure, such as valvular heart disease, hypertensive heart disease, or complete left bundle block, were excluded from this study, resulting in a sample size of 22 patients with AIHF (age, $70 \pm 12 \mathrm{y} ; 16$ men and 6 women). The study protocol was received and approved by the institutional review board, and written informed consent was obtained from all study participants. 


\section{Myocardial Perfusion SPECT}

All patients in this study underwent both nicorandil-stress and rest myocardial perfusion SPECT at a minimum interval of $48 \mathrm{~h}$. Test orders were randomized to ensure that $50 \%$ of participants underwent the nicorandil-stress test first, while the remainder underwent the rest test first. The acquisition was performed using the dual-head $\gamma$-camera Infinia (GE Healthcare). For nicorandil-stress imaging, $600 \mathrm{MBq}$ of ${ }^{99 \mathrm{~m}} \mathrm{Tc}$-sestamibi were injected immediately after a $0.2 \mathrm{mg} / \mathrm{kg}$ nicorandil bolus injection, which was administered over a 5-min period, while SPECT data were acquired $30 \mathrm{~min}$ later. For rest imaging, SPECT data were acquired $30 \mathrm{~min}$ after injecting $600 \mathrm{MBq}$ of ${ }^{99 \mathrm{~m} T c} \mathrm{~T}-$ sestamibi. In both tests, 30 projection images were obtained in $6^{\circ}$ increments over an orbit of $180^{\circ}$ at a rate of 45 beats per projection using electrocardiogram-gated acquisition. The image matrix size was 64 , and a low-energy high-resolution collimator was used. Collected data were reformatted into nonelectrocardiogram-gated short axial, horizontal-long axial, and vertical-long axial SPECT images via reconstruction with ordered-subsets expectation maximization and without attenuation correction.

\section{Data Acquisition}

For both nicorandil-stress and rest images, the left ventricular myocardium was divided into 17 segments (21), and 2-dimensional regions of interest (ROIs) were drawn on each myocardial segment in the short axial, horizontal-long axial, and vertical-long axial SPECT images. ROIs were drawn on the upper mediastinum in transaxial SPECT images as background to facilitate the calculation of myocardiumto-background ratio (MBR = peak value of the left ventricular myocardial segments/mean value of the upper mediastinum). Both the mean MBR of all segments and the MBR-IR (MBR-IR $=$ MBR $_{\text {stress }} l$ $M_{B R} R_{\text {rest }}$ ) were calculated for all patients using this method. In order to evaluate the clinical importance of MBR-IR, patients in this study were divided into 2 groups based on their MBR-IR value. The optimal cutoff MBR-IR value was determined using a receiver-operatingcharacteristic curve in reference to major adverse cardiac event (MACE) occurrences.

\section{Evaluation of Prognosis}

All patients were observed over $5 \mathrm{y}$ from the onset of AIHF and for the occurrence of MACE, which were defined as fatal cardiac events, nonfatal myocardial infarctions, revascularization procedures due to exacerbation of angina pectoris, or hospitalizations due to the deterioration of heart failure. The endpoint for this study was defined as either the occurrence of MACE or $5 \mathrm{y}$ after AIHF onset. Events that occurred within $30 \mathrm{~d}$ of initial onset were not considered as MACE. The relationship between the occurrence of MACE and various clinical parameters, including MBR-IR, was analyzed.

\section{Statistical Analysis}

Continuous variables are expressed as mean \pm SD for normally distributed data. Data not distributed normally, such as nicorandilstress and rest MBR and MBR-IR, are presented as medians with 25 th and 75 th percentiles. Categorical variables are presented as counts (\%).

A paired $t$ test was used to compare cardiovascular parameters, such as blood pressure and heart rate under nicorandil-stress and rest conditions. Left ventricular ejection fraction (LVEF), left ventricular end-diastolic volume (LVEDV), left ventricular end-systolic volume (LVESV), summed stress score (SSS), summed rest score (SRS), and both nicorandil-stress and rest MBR and MBR-IR were not normally distributed and were compared using the Wilcoxon signed-rank test or Mann-Whitney $U$ test. Categorical variables were compared using the Fisher's exact probability test for the bivariate data and the MannWhitney $U$ test for New York Heart Association class. Receiveroperating-characteristic analysis was used to determine the optimal cutoff value of MBR-IR for predicting MACE occurrences by maximizing the sum of sensitivity and specificity. The proportion of event-free patients was estimated by the Kaplan-Meier method and compared between high- and low-MBR-IR groups using the log-rank test. Variables with a significance level of $P$ less than 0.2 in the univariate Cox regression analysis were included in a multivariate Cox regression model to evaluate factors independently associated with the future occurrence of MACE.

A $P$ value of less than 0.05 was considered statistically significant. All statistical analyses were performed using StatMate IV software (version 4.01; Advanced Technology for Medicine and Science).

\section{RESULTS}

Clinical Characteristics and Cardiovascular Parameter Variation Between Nicorandil-Stress and Rest Conditions

Twenty-two patients (age, $70 \pm 12 \mathrm{y} ; 16$ men, 6 women) underwent both nicorandil-stress and rest myocardial perfusion SPECT for the assessment of MBR-IR. Patient characteristics, including comorbidities and intervals between the nicorandilstress test, the rest test, and CAG, are presented in Table 1. Patients underwent the first myocardial perfusion SPECT once their clinical condition was considered stable, 8 (6-12) days after the initial onset of AIHF. Table 2 shows cardiovascular parameter variations between nicorandil-stress and rest test conditions. Although systolic and diastolic blood pressure decreased significantly and heart rate increased significantly under nicorandil-stress conditions compared with the values at rest conditions $(P<0.001, P=0.004$, and $P<0.001)$, both blood pressure and heart rate were within a safe range and no serious adverse events occurred after intravenous nicorandil administration. No significant changes were observed in LVEF, LVEDV, and LVESV between nicorandil-stress and rest conditions $(P=0.106,0.516$, and 0.276 , respectively).

\section{Patient Categorization by MBR-IR Value}

Using receiver-operating-characteristic analysis for predicting MACE occurrences, 11 patients were assigned to the highMBR-IR group, while the remaining 11 were assigned to the lowMBR-IR group. Median MBR-IR was 1.55 (1.34-1.61) in the high group and $1.08(1.02-1.10)$ in the low group (Table 3$)$. The cutoff value for MBR-IR was 1.15 , and the area under the curve, sensitivity, and specificity were $0.866,91 \%$, and $91 \%$, respectively.

\section{Patient Prognosis}

All participants were observed over $5 \mathrm{y}$ from the onset of AIHF. Of the 22 subjects, 12 (55\%) experienced MACE during the 5-y follow-up period. A fatal cardiac event occurred in 1 patient, a nonfatal myocardial infarction in 1 patient, revascularization due to exacerbation of angina pectoris in 2 patients, and hospitalization due to deterioration of heart failure in 8 patients. Of the 12 incidents of MACE, only a single case of deterioration of heart failure and a single fatal cardiac event occurred in the high-MBRIR group. In summary, the proportion of patients who experienced MACE was significantly higher in the low-MBR-IR group than in the high-MBR-IR group $(10 / 11$ vs. $2 / 11$ patients, $P<0.001)$ (Fig. 1). A comparison of the clinical profiles of all subjects who did and did not experience MACE is presented in Table 4. Table 5 shows the results of the univariate and multivariate Cox regression analysis for the occurrence of MACE. In the multivariate analysis, 
TABLE 1

Patient Characteristics

\begin{tabular}{|cc}
\hline \multicolumn{1}{c}{ Characteristic } & $n$ or mean \pm SD \\
\hline No. of patients & 22 \\
\hline Age $(\mathrm{y})$ & $70 \pm 12$ \\
\hline Male & $16(73 \%)$ \\
\hline NYHA class (I/II/III/IV) & $0 / 2 / 2 / 18$ \\
\hline BNP (pg/mL) & $524(258-744)$ \\
\hline Coronary risk factors & \\
\hline Hypertension & $21(95 \%)$ \\
\hline Dyslipidemia & $15(68 \%)$ \\
\hline Diabetes mellitus & $13(59 \%)$ \\
\hline Interval (d) & \\
\hline Onset to first MPI & $8(6-12)$ \\
\hline Nicorandil-stress and rest & $3(3-5)$ \\
\hline Onset to CAG & $7(4-15)$ \\
\hline Previous MI & $20(91 \%)$ \\
\hline IVD/2VD/3VD & $2 / 7 / 13$ \\
\hline
\end{tabular}

NYHA $=$ New York Heart Association; BNP = brain natriuretic peptide; $\mathrm{MPI}=$ myocardial perfusion imaging; $\mathrm{MI}=$ myocardial infarction; VD = vessel disease.

Categorical data are expressed as numbers, followed by percentages in parentheses, unless otherwise indicated; continuous data are expressed as mean \pm SD or median (25th-75th).

low MBR-IR was determined to be the sole independent prognostic factor $(P=0.004)$.

\section{Case Presentations}

Figure 2 shows a typical case of a patient in the high-MBR-IR group. This 77-y-old man showed ST depression in $\mathrm{V}_{1}-\mathrm{V}_{5}$ on the electrocardiogram on admission. Emergency CAG showed $75 \%$ stenosis in the left main coronary artery and the proximal left circumflex artery and chronic total occlusion in the proximal left anterior descending artery. Eight days after onset, a nicorandil-stress test was performed, and rest imaging was performed $3 \mathrm{~d}$ later. The rest image showed anteroseptal-apical perfusion defects, whereas improved perfusion was observed for the anteroseptal wall in the nicorandilstress image. MBR-IR was relatively high for an AIHF patient with a value of 1.48. In this case, the patient remained MACE-free for the entire 5-y follow-up period.

Figure 3 shows a typical case of a patient in the low-MBR-IR group who experienced MACE during the follow-up period. In this 69-y-old woman, ST depression in $\mathrm{V}_{1}-\mathrm{V}_{4}$ was observed on electrocardiogram on admission. Emergency CAG showed $75 \%$ stenosis of the proximal left anterior descending artery, first diagonal branch, obtuse marginal branch, and posterolateral artery. Six days after onset, a nicorandil-stress test was performed, and rest imaging was performed 2 d later. Nicorandil-stress and rest images showed anteroseptal-apical perfusion defects, and perfusion improvement was observed only in the marginal zone of the previous myocardial infarction in the nicorandil-stress image.
MBR-IR was low, with a value of 1.09 . In this case, the patient was hospitalized due to deterioration of heart failure $189 \mathrm{~d}$ after initial onset.

\section{DISCUSSION}

In the present study, to assess the effect of nicorandil, MBR-IR as an alternative to MFR was calculated using conventional myocardial perfusion SPECT with intravenous administration of nicorandil and the prognostic value of MBR-IR was evaluated in patients with AIHF. The results showed that MBR-IR was a valuable prognostic marker, with low MBR-IR indicating a poorer prognosis than high MBR-IR. No other parameters proved to be of prognostic value within this patient group.

\section{Myocardial Perfusion SPECT with Intravenous Bolus Administration of Nicorandil}

Using a vasodilator, such as adenosine, has been standard for calculating MFR and FFR in a clinical setting (15). Adenosine is a standard drug for achieving maximum hyperemia, mostly in coronary microarteries, by activating A2A receptors in vascular smooth muscles (11). However, the use of such agents is limited in patients with AIHF because of the potential for complications, such as chest discomfort; bronchial asthma; hypotension; arrhythmia, including transient atrioventricular block; and myocardial ischemia due to coronary steal phenomenon. Nicorandil is a particularly effective hyperemic agent, as it induces dilatation of microarteries as well as pericardial arteries unlike adenosine, and this response is more significant in ischemic areas (12). Figures 2 and 3 show the effects of nicorandil on ischemic tissue surrounding the necrotic core of a previous myocardial infarction. Unlike adenosine-stress images, myocardial perfusion in the ischemic area is improved in the nicorandil-stress images. A recent study reported nicorandil to be a safer and more effective vasodilator than ATP for the measurement of FFR $(16,17)$. As nicorandil has both nitratelike and ATP-sensitive potassium-channel-activating properties, it does not cause coronary steal phenomenon (11). Furthermore, despite differing modes of action, a strong, linear correlation between FFR using ATP and FFR using nicorandil has been demonstrated

\section{TABLE 2}

Cardiovascular Parameter Variations Between Nicorandil-Stress and Rest Conditions

\begin{tabular}{lccc}
\hline $\begin{array}{c}\text { Cardiovascular } \\
\text { parameter }\end{array}$ & $\begin{array}{c}\text { Nicorandil- } \\
\text { stress }\end{array}$ & Rest & $P$ \\
\hline $\begin{array}{c}\text { Systolic BP } \\
(\mathrm{mm} \mathrm{Hg})\end{array}$ & $98 \pm 16$ & $123 \pm 15$ & $<0.001$ \\
$\begin{array}{c}\text { Diastolic BP } \\
(\mathrm{mm} \mathrm{Hg})\end{array}$ & $60 \pm 6$ & $70 \pm 15$ & 0.004 \\
\hline Heart rate $(\mathrm{bpm})$ & $78 \pm 15$ & $67 \pm 11$ & $<0.001$ \\
\hline LVEF $(\%)$ & $40(31-44)$ & $36(30-40)$ & 0.106 \\
LVEDV (mL) & $154(118-191)$ & $161(105-196)$ & 0.516 \\
\hline LVESV (mL) & $96(71-128)$ & $108(63-131)$ & 0.276 \\
\hline & & & \\
\hline BP $=$ blood pressure. \\
Data are expressed as mean \pm SD or median (25th-75th).
\end{tabular}


TABLE 3

Comparison of Clinical Profiles Between High- and Low-MBR-IR Groups

\begin{tabular}{|c|c|c|c|}
\hline Characteristic & High MBR-IR $(n=11[50 \%])$ & Low MBR-IR $(n=11[50 \%])$ & $P$ \\
\hline Age $(y)$ & $75 \pm 9$ & $65 \pm 13$ & 0.046 \\
\hline Male & $7(64 \%)$ & $9(82 \%)$ & 0.635 \\
\hline NYHA class & $0 / 0 / 2 / 9$ & $0 / 2 / 0 / 9$ & 0.883 \\
\hline $\mathrm{BNP}(\mathrm{pg} / \mathrm{mL})$ & $534(189-965)$ & $513(412-704)$ & 0.844 \\
\hline Hypertension & $10(91 \%)$ & $11(100 \%)$ & 1.000 \\
\hline Dyslipidemia & $6(55 \%)$ & $9(82 \%)$ & 0.361 \\
\hline Diabetes mellitus & $5(45 \%)$ & $8(73 \%)$ & 0.387 \\
\hline Previous myocardial infarction & $10(91 \%)$ & $10(91 \%)$ & 1.000 \\
\hline SSS & $17(15-24)$ & 25 (23-28) & 0.139 \\
\hline SRS & $19(16-28)$ & 27 (24-29) & 0.144 \\
\hline Rest LVEF (\%) & $38(32-53)$ & $31(30-38)$ & 0.216 \\
\hline Rest LVEDV (mL) & $136(90-165)$ & $186(156-201)$ & 0.111 \\
\hline Rest LVESV (mL) & $83(43-116)$ & $118(103-140)$ & 0.102 \\
\hline Nicorandil-stress MBR & $29.49(26.57-37.44)$ & $26.70(16.96-31.93)$ & 0.151 \\
\hline Rest MBR & $18.62(16.62-26.80)$ & $25.39(16.17-30.71)$ & 0.507 \\
\hline MBR-IR & $1.55(1.34-1.61)$ & $1.08(1.02-1.10)$ & $<0.001$ \\
\hline MBR-IR in infarcted region & $1.06(1.00-1.06)$ & $0.98(0.97-1.00)$ & 0.149 \\
\hline MBR-IR in ischemic region & $1.57(1.41-1.74)$ & $1.18(1.16-1.22)$ & 0.002 \\
\hline
\end{tabular}

$\mathrm{NYHA}=$ New York Heart Association; BNP = brain natriuretic peptide; SSS $=$ summed stress score; SRS $=$ summed rest score.

Categorical data are expressed as counts (\%), and continuous data are expressed as mean \pm SD or median (25th-75th).

(17). Thus, nicorandil was chosen as the vasodilator for the present study, and its therapeutic effect was also demonstrated.

\section{Calculating MBR-IR Using Conventional Myocardial SPECT}

Traditionally, MFR is calculated using myocardial perfusion PET/CT and intravenous administration of vasodilators commonly used in myocardial perfusion imaging, including adenosine $(18,19)$. MFR is calculated by dividing vasodilator stress MBF by rest MBF, as obtained by dynamic acquisition. However, as PET/CT is available only at a limited number of facilities, a more convenient method for calculating MFR has long been sought. MBF-IR, determined using myocardial perfusion SPECT, could provide an alternative for MFR (20). However, as is the case with PET/ $\mathrm{CT}$, this method still requires dynamic acquisition that may lead to inaccurate results due to low acquisition efficiency associated with SPECT. In the present study, a simpler and more convenient method for calculating a prognostic marker, MBRIR, another alternative to MFR, using static SPECT images was devised, thus removing the need for dynamic acquisition. As SPECT is usually much more accessible than PET/CT, MBRIR can be used in a large number of facilities. MBR was calculated by dividing peak value of the left ventricular myocardial segments by mean value of the upper mediastinum. MBR-IR was calculated by dividing stress MBR by rest MBR. As the median stress-to-rest ratio of mean background counts in mediastinum was $1.00(0.92-1.11)$ and fluctuations were low, MBRIR was similar to the stress-to-rest ratio of mean myocardial accumulation. In several cases with severe AIHF, high pulmonary accumulation may affect mediastinal counts. However, this adverse effect was minimized by placing mediastinal ROIs as distant from the lungs as possible.

\section{Comparing MBR-IR and MFR in Patients with AlHF}

The reference range of MFR derived from myocardial perfusion PET/CT was previously reported to be $2.74 \pm 0.67$, whereas the mean value of MFR was $1.23 \pm 0.43$ in severe ischemic areas $(22,23)$. In patients with suspected myocardial ischemia, MBF-IR

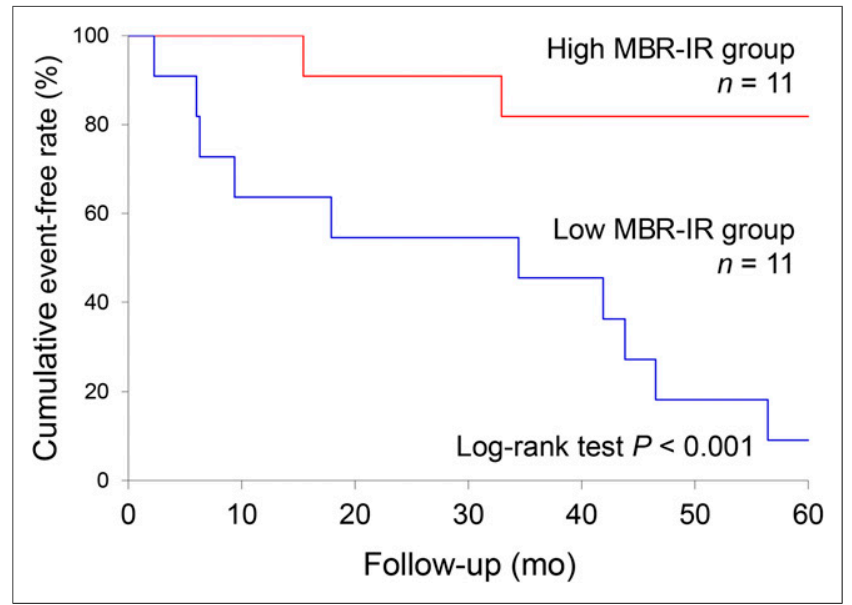

FIGURE 1. Kaplan-Meier curve in reference to MACE stratified by MBR-IR value $y$-axis represents cumulative event-free rate (log-rank test, $P<0.001$ ). 
TABLE 4

Comparison of Clinical Profiles of All Patients Who Did and Did Not Experience MACE

\begin{tabular}{|c|c|c|c|}
\hline Characteristic & $\operatorname{MACE}(n=12[55 \%])$ & No MACE $(n=10$ [45\%]) & $P$ \\
\hline Age $(y)$ & $70 \pm 10$ & $71 \pm 14$ & 0.950 \\
\hline Male & $9(75 \%)$ & 7 (70\%) & 1.000 \\
\hline NYHA class & $0 / 0 / 1 / 11$ & $0 / 2 / 1 / 7$ & 0.185 \\
\hline $\mathrm{BNP}(\mathrm{pg} / \mathrm{mL})$ & $664(420-794)$ & $408(184-687)$ & 0.277 \\
\hline Hypertension & $12(100 \%)$ & 9 (90\%) & 0.455 \\
\hline Dyslipidemia & $9(75 \%)$ & $6(60 \%)$ & 0.652 \\
\hline Diabetes mellitus & $7(58 \%)$ & $6(60 \%)$ & 1.000 \\
\hline Previous myocardial infarction & $12(100 \%)$ & $8(80 \%)$ & 0.195 \\
\hline SSS & $25(21-28)$ & $20(15-25)$ & 0.209 \\
\hline SRS & 27 (23-29) & $22(15-29)$ & 0.427 \\
\hline Low MBR-IR & $10(83 \%)$ & $1(10 \%)$ & 0.002 \\
\hline Rest LVEF (\%) & $31(30-39)$ & $38(31-53)$ & 0.477 \\
\hline \multicolumn{4}{|c|}{ Treatments after myocardial perfusion imaging } \\
\hline $\mathrm{PCl}$ & $3(25 \%)$ & $1(10 \%)$ & 0.594 \\
\hline CABG & $1(8 \%)$ & $1(10 \%)$ & 1.000 \\
\hline Nicorandil & $7(58 \%)$ & $5(50 \%)$ & 1.000 \\
\hline ARB & $11(92 \%)$ & $6(60 \%)$ & 0.135 \\
\hline$\beta$-blocker & $10(83 \%)$ & $9(90 \%)$ & 1.000 \\
\hline Statin & $10(83 \%)$ & $6(60 \%)$ & 0.348 \\
\hline Pacemaker implantation & $2(17 \%)$ & $0(0 \%)$ & 0.481 \\
\hline ICD implantation & $1(8 \%)$ & $0(0 \%)$ & 1.000 \\
\hline
\end{tabular}

NYHA = New York Heart Association; BNP = brain natriuretic peptide; SSS = summed stress score; SRS = summed rest score; PCI = percutaneous coronary intervention; $\mathrm{CABG}=$ coronary artery bypass grafting; $\mathrm{ARB}=$ angiotensin receptor blocker; ICD = implantable cardioverter-defibrillator.

Categorical data are expressed as counts (\%), and continuous data are expressed as mean \pm SD or median (25th-75th).

in low-, moderate-, and high-CFR groups were $1.19 \pm 0.19,1.50 \pm$ 0.18 , and $1.78 \pm 0.18$, respectively, and MBF-IR closely correlated with CFR (20). As AIHF is generally considered a severe ischemic heart disease, CFR in such patients is likely to be low. In this study, MBR-IR was 1.55 (1.34-1.61) in the high-MBR-IR group and $1.08(1.02-1.10)$ in the low-MBR-IR group. These figures equate well with the aforementioned MBF-IR values in moderate- and low-MBF-IR groups. Furthermore, whereas MFR and CFR are measurements of blood flow, MBR-IR is a count ratio of myocardial accumulation. Although SPECT has low sensitivity in diagnosing multivessel disease, MBR-IR could be instrumental for the detection of diffused myocardial ischemia. Because of the low extraction fraction of ${ }^{99 \mathrm{~m}} \mathrm{Tc}$-sestamibi into myocardium, the MBR-IR may underestimate the CFR in highly perfused myocardium similarly to MBF-IR (20). However, as MFR in patients with AIHF is commonly low, the MBR-IR in this study population can be an alternative to MFR.

\section{Prognostic Value of MBR-IR for MACE}

Patient prognosis in cases with low MFR is poor, even in the absence of myocardial perfusion defects $(18,24)$. In the present study, 12 of $22(55 \%)$ patients with AIHF experienced MACE within the 5-y follow-up period. Of these, 10 patients were from the low-MBR-IR group (83\%), whereas only 2 patients were from the high-MBR-IR group (17\%). Ziadi et al. reported that patients with abnormal MFR experienced MACE significantly more than the other groups within the first follow-up year (24\%) (18). The proportion of MACE occurrence in this study was $18 \%$ in the first year, a value identical to the report mentioned above. Slart et al. reported that patients with low MFR $(<1.34)$ had the poorest outcomes compared with the 2 other groups (MFR $>1.69$ and 1.34-1.69) (25). Similarly, the results from this study showed that patients with low MBR-IR have poorer prognoses than patients with high MBR-IR. In summary, MBR-IR derived from nicorandilstress myocardial perfusion SPECT appears to have a high prognostic value for MACE, similarly to MFR derived from myocardial perfusion PET/CT.

The main limitation of this study was the small patient sample size of 22. Statistical reliability is thus limited. Although the patient parameters other than MBR-IR were not found to have prognostic value in this study, it is widely accepted that factors such as diabetes mellitus, previous myocardial infarction, and SSS are associated with MACE $(26,27)$. However, because low MBR-IR was the sole independent factor associated with MACE occurrence, we may infer that low MBR-IR is indeed a strong predictor for MACE. A further limitation in the study is the lack of both a control group and a group with mild coronary artery disease. As a result, the value of MBR-IR in healthy patients is unknown, as is the predictive value of 
TABLE 5

Univariate and Multivariate Cox Regression Analysis for Occurrence of MACE

\begin{tabular}{|c|c|c|c|c|c|c|}
\hline \multirow[b]{2}{*}{ Characteristic } & \multicolumn{3}{|c|}{ Univariate } & \multicolumn{3}{|c|}{ Multivariate } \\
\hline & Hazard ratio & $95 \%$ confidence interval & $P$ & Hazard ratio & $95 \%$ confidence interval & $P$ \\
\hline Age & 0.999 & $0.967-1.032$ & 0.945 & & & \\
\hline NYHA class IV & 1.589 & $0.536-4.312$ & 0.404 & & & \\
\hline BNP & 1.000 & $0.999-1.001$ & 0.160 & 1.001 & $0.999-1.002$ & 0.086 \\
\hline Hypertension & 1.833 & $0.246-13.677$ & 0.554 & & & \\
\hline Dyslipidemia & 1.259 & $0.512-3.097$ & 0.615 & & & \\
\hline Diabetes mellitus & 0.947 & $0.404-2.220$ & 0.901 & & & \\
\hline Previous myocardial infarction & 1.920 & $0.446-8.261$ & 0.381 & & & \\
\hline SSS & 1.009 & $0.966-1.055$ & 0.683 & & & \\
\hline SRS & 1.003 & $0.964-1.044$ & 0.866 & & & \\
\hline Low MBR-IR & 3.640 & $1.456-9.103$ & 0.006 & 3.913 & $1.552-9.868$ & 0.004 \\
\hline Rest LVEF & 0.992 & $0.964-1.020$ & 0.576 & & & \\
\hline \multicolumn{7}{|l|}{ Treatment after MPI } \\
\hline $\mathrm{PCl}$ & 1.601 & $0.538-4.761$ & 0.398 & & & \\
\hline CABG & 0.771 & $0.179-3.309$ & 0.726 & & & \\
\hline Nicorandil & 1.091 & $0.471-2.529$ & 0.839 & & & \\
\hline ARB & 1.639 & $0.598-4.495$ & 0.337 & & & \\
\hline$\beta$-blocker & 1.013 & $0.298-3.451$ & 0.983 & & & \\
\hline Statin & 1.359 & $0.528-3.501$ & 0.525 & & & \\
\hline
\end{tabular}

NYHA = New York Heart Association; BNP = brain natriuretic peptide; SSS = summed stress score; SRS = summed rest score; $\mathrm{MPI}=$ myocardial perfusion imaging; $\mathrm{PCl}=$ percutaneous coronary intervention; $\mathrm{CABG}=$ coronary artery bypass grafting; $\mathrm{ARB}=$ angiotensin receptor blocker; ICD = implantable cardioverter-defibrillator.

MBR-IR in patients with milder coronary artery disease. Nonetheless, it may be speculated that the lower limit for healthy MBR-IR is similar to that of MBF-IR (20). In this study, rest and nicorandil-stress tests were conducted on separate days to ensure complete tracer elimination for each test. However, this interval may have allowed for fluctuation in the patients' clinical condition, although the order

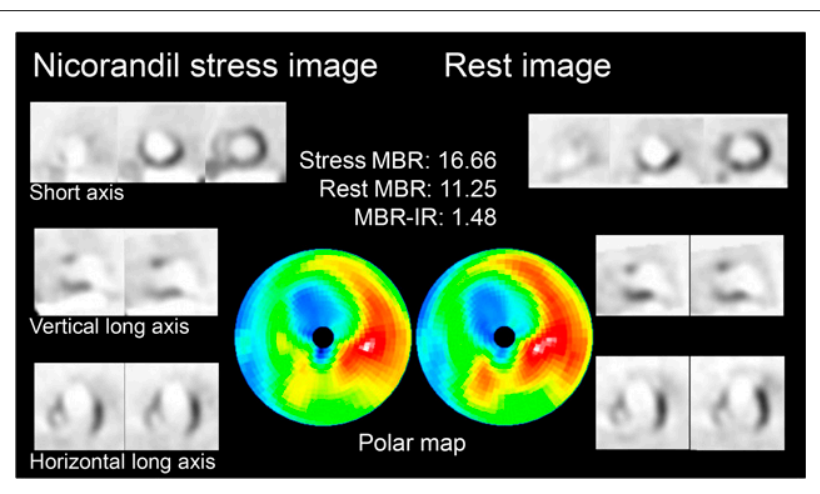

FIGURE 2. A 77-y-old man with acute ischemic heart failure in high-MBRIR group. Rest myocardial perfusion SPECT images show anteroseptalapical and distal inferior perfusion defects, and perfusion improvement is observed for anteroseptal wall in nicorandil-stress images, excluding central area of previous myocardial infarction site. MBR-IR is relatively high for an AlHF patient, with a value of 1.48. This patient remained MACE-free for entire 5-y follow-up period. that the tests were conducted in was randomized to reduce the influence of such changes. Usually, rest and stress myocardial perfusion SPECT tests using vasodilators are performed in a single day. This MBR-IR calculation could also be applied to the conventional stress myocardial perfusion SPECT. We believe this method is instrumental for detecting multivessel coronary artery disease.

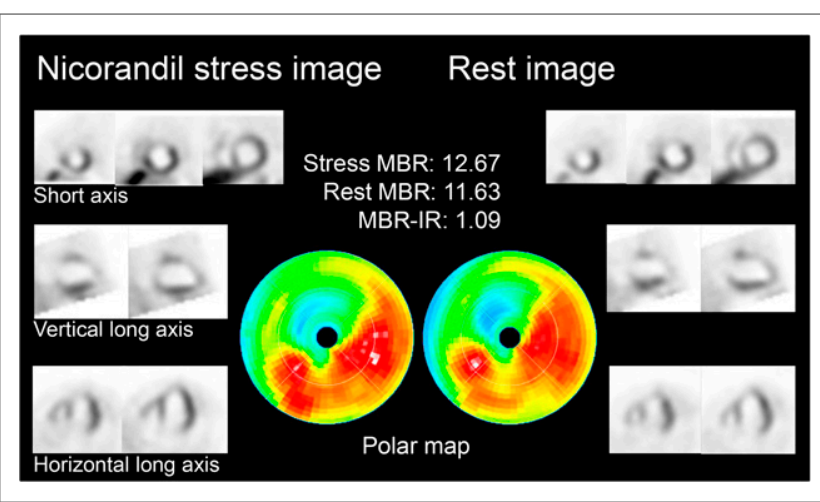

FIGURE 3. A 69-y-old woman with acute ischemic heart failure in lowMBR-IR group. Nicorandil stress and rest myocardial perfusion SPECT images show anteroseptal-apical perfusion defects, and perfusion improvement is observed only in marginal zone of previous myocardial infarction site in nicorandil-stress images. MBR-IR is low, with a value of 1.09. This patient was hospitalized due to deterioration of heart failure $189 \mathrm{~d}$ after initial onset. 


\section{CONCLUSION}

In this study, the effect of nicorandil was estimated by calculating MBR-IR, a value more easily obtainable than MFR, using nicorandil-stress myocardial perfusion SPECT in patients with AIHF. Furthermore, the MBR-IR may have a high prognostic value for MACE in patients with AIHF. A novel method for predicting CFR was used and has the potential to be used in a wider clinical setting than MFR, thus making accurate prognosis more accessible.

\section{DISCLOSURE}

The costs of publication of this article were defrayed in part by the payment of page charges. Therefore, and solely to indicate this fact, this article is hereby marked "advertisement" in accordance with 18 USC section 1734 . No potential conflict of interest relevant to this article was reported.

\section{ACKNOWLEDGMENTS}

We are grateful to the radiology technologists Kyoji Asano, Toyohiko Yanagawa, and Toshio Maki for their technical assistance in the administration of myocardial perfusion SPECT.

\section{REFERENCES}

1. Flaherty JD, Bax JJ, De Luca L, et al. Acute heart failure syndromes in patients with coronary artery disease early assessment and treatment. J Am Coll Cardiol. 2009;53:254-263.

2. Beller GA. More evidence for the survival benefit of coronary revascularization versus medical therapy in patients with ischemic cardiomyopathy and hibernating myocardium. Circ Cardiovasc Imaging. 2013;6:355-357.

3. Christenson JT, Schmuziger M, Simonet F. Reoperative coronary artery bypass procedures: risk factors for early mortality and late survival. Eur J Cardiothorac Surg. 1997;11:129-133.

4. JCS Joint Working Group. Guidelines for treatment of acute heart failure (JCS 2011). Circ J. 2013;77:2157-2201.

5. Yancy CW, Jessup M, Bozkurt B, et al. 2013 ACCF/AHA guideline for the management of heart failure: executive summary: a report of the American College of Cardiology Foundation/American Heart Association Task Force on practice guidelines. Circulation. 2013;128:1810-1852.

6. McMurray JJ, Adamopoulos S, Anker SD, et al. ESC guidelines for the diagnosis and treatment of acute and chronic heart failure 2012: The Task Force for the Diagnosis and Treatment of Acute and Chronic Heart Failure 2012 of the European Society of Cardiology. Developed in collaboration with the Heart Failure Association (HFA) of the ESC. Eur Heart J. 2012;33:1787-1847.

7. Metra M, Teerlink JR, Voors AA, et al. Vasodilators in the treatment of acute heart failure: what we know, what we don't. Heart Fail Rev. 2009;14:299-307.

8. Tanaka K, Kato K, Takano T, et al. Acute effects of intravenous nicorandil on hemodynamics in patients hospitalized with acute decompensated heart failure. J Cardiol. 2010;56:291-299.

9. Kato K, Takano T, Asanoi E. Acute effect of a bolus intravenous administration of nicorandil (Sigmart) in patients with acute heart failure or acute exacerbation of chronic heart failure. Jpn Pharmacol Ther. 2008;36:s25-s34.
10. Akai K, Wang Y, Sato K, et al. Vasodilatory effect of nicorandil on coronary arterial microvessels: its independency on vessel size and the involvement of the ATP-sensitive potassium channels. J Cardiovasc Pharmacol. 1995;26:541-547.

11. Komaru T, Kanatsuka H, Shirato K. Coronary microcirculation: physiology and pharmacology. Pharmacol Ther. 2000;86:217-261.

12. Markham A, Plosker GL, Goa KL. Nicorandil. An updated review of its use in ischaemic heart disease with emphasis on its cardioprotective effects. Drugs. 2000;60:955-974.

13. Okamura A, Rakugi H, Ohishi M, et al. Additive effects of nicorandil on coronary blood flow during continuous administration of nitroglycerin. J Am Coll Cardiol. 2001;37:719-725.

14. Giles TD, Pina IL, Quiroz AC, et al. Hemodynamic and neurohumoral responses to intravenous nicorandil in congestive heart failure in humans. J Cardiovasc Pharmacol. 1992;20:572-578.

15. De Bruyne B, Pijls NH, Heyndrickx GR, Hodeige D, Kirkeeide R, Gould KL. Pressure-derived fractional flow reserve to assess serial epicardial stenoses: theoretical basis and animal validation. Circulation. 2000;101:1840-1847.

16. Jang HJ, Koo BK, Lee HS, et al. Safety and efficacy of a novel hyperaemic agent, intracoronary nicorandil, for invasive physiological assessments in the cardiac catheterization laboratory. Eur Heart J. 2013;34:2055-2062.

17. Kato D, Takashima H, Waseda K, et al. Feasibility and safety of intracoronary nicorandil infusion as a novel hyperemic agent for fractional flow reserve measurements. Heart Vessels. 2015;30:477-483.

18. Ziadi MC, Dekemp RA, Williams KA, et al. Impaired myocardial flow reserve on rubidium-82 positron emission tomography imaging predicts adverse outcomes in patients assessed for myocardial ischemia. J Am Coll Cardiol. 2011;58: $740-748$.

19. Fiechter M, Ghadri JR, Gebhard C, et al. Diagnostic value of ${ }^{13} \mathrm{~N}$-ammonia myocardial perfusion PET: added value of myocardial flow reserve. $\mathrm{J} \mathrm{Nucl}$ Med. 2012;53:1230-1234.

20. Taki J, Fujino S, Nakajima K, et al. ${ }^{99 \mathrm{~m} T c-s e s t a m i b i}$ retention characteristics during pharmacologic hyperemia in human myocardium: comparison with coronary flow reserve measured by Doppler flowire. J Nucl Med. 2001;42:14571463.

21. Cerqueira MD, Weissman NJ, Dilsizian V, et al. Standardized myocardial segmentation and nomenclature for tomographic imaging of the heart: a statement for healthcare professionals from the Cardiac Imaging Committee of the Council on Clinical Cardiology of the American Heart Association. Int J Cardiovasc Imaging. 2002;18:539-542.

22. Renaud JM, DaSilva JN, Beanlands RS, DeKemp RA. Characterizing the normal range of myocardial blood flow with ${ }^{82}$ rubidium and ${ }^{13} \mathrm{~N}$-ammonia PET imaging. J Nucl Cardiol. 2013;20:578-591.

23. Zhang X, Schindler TH, Prior JO, et al. Blood flow, flow reserve, and glucose utilization in viable and nonviable myocardium in patients with ischemic cardiomyopathy. Eur J Nucl Med Mol Imaging. 2013;40:532-541.

24. Fukushima K, Javadi MS, Higuchi T, et al. Prediction of short-term cardiovascular events using quantification of global myocardial flow reserve in patients referred for clinical ${ }^{82} \mathrm{Rb}$ PET perfusion imaging. J Nucl Med. 2011;52:726-732.

25. Slart RH, Zeebregts CJ, Hillege HL, et al. Myocardial perfusion reserve after a PET-driven revascularization procedure: a strong prognostic factor. $\mathrm{J} \mathrm{Nucl} \mathrm{Med}$. 2011;52:873-879.

26. Haffner SM, Lehto S, Ronnemaa T, Pyorala K, Laakso M. Mortality from coronary heart disease in subjects with type 2 diabetes and in nondiabetic subjects with and without prior myocardial infarction. N Engl J Med. 1998;339:229-234.

27. Hachamovitch R, Berman DS, Shaw LJ, et al. Incremental prognostic value of myocardial perfusion single photon emission computed tomography for the prediction of cardiac death differential stratification for risk of cardiac death and myocardial infarction. Circulation. 1998;97:535-543. 